\title{
Patient aggression in psychiatric services: the experience of a sample of nurses at two psychiatric facilities in Nigeria
}

\author{
BO James', EW Isa', N Oud ${ }^{2}$ \\ 1Department of Clinical Services, Federal Psychiatric Hospital, Uselu, Benin-City, Edo State, Nigeria \\ 2Oud Consultancy, Amsterdam, the Netherlands
}

\begin{abstract}
Objective: Aggression is a common feature in psychiatric in-patient units in Africa. The attitudes of psychiatric nurses and their perceptions of the frequency of in-patient aggression have not been explored in the Nigerian context. Method: Using a crosssectional study design, two self-report questionnaires (the Attitudes toward Aggression Scale (ATAS) and the Perception of the Prevalence of Aggression Scale (POPAS)) were administered to nursing staff ( $\mathrm{n}=73$ ) at two psychiatric facilities in Benin City, Nigeria. Results: Overall, nurses viewed aggression as offensive, destructive and intrusive. They were less likely to view it as a means of communication or serving protective functions. Verbal aggression was the commonest type of aggression experienced while sexual intimidation and suicide attempts were least common. Male nurses were more likely to experience physical violence and aggressive 'splitting' behaviours, while nurses with over a decade of professional experience were more likely to experience verbal and humiliating aggressive behaviours. In contrast to previous studies, fewer nurses required days off work due to aggressive behaviour. Conclusion: Aggression is commonly experienced by nurses in in-patient units in Nigeria. Their views were predominantly negative. Training programmes are required to change staff attitudes as well as research on the cultural factors mediating these attitude dispositions.
\end{abstract}

Key words: Nurses; Aggression; Attitudes; Nigeria

Received: $25-03-2010$

Accepted: 07-05-2010

doi: 10.4314/ajpsy.v14i2.4

\section{Introduction}

Aggression within in-patient psychiatric settings is well researched, and the attitude of health professionals towards aggression is often the focus of many research reports. ${ }^{1-4}$ Attitudes are defined as 'a predisposition toward any person, idea or object and contains cognitive, affective and behavioural components. ${ }^{5}$ Attitudes towards aggression has three components. The first is the cognitive component,

\section{Correspondence}

Dr. BO James

Department of Clinical Services, Federal Psychiatric Hospital, Uselu,

Benin City, Nigeria

email: bawojames@yahoo.com which comprises the degree of exposure to episodes or acts of aggression, the nature or precipitants of aggressive behaviour, the management of outcomes, and severity of injuries sustained. ${ }^{3}$ The second and third components are the affective and behavioural components and these include thoughts, beliefs and overt acts directed toward another person or group of persons. ${ }^{6}$ Nurses working in mental health settings frequently experience acts of aggression from in-patients. ${ }^{7-8}$ The frequency depends on the treatment setting (higher in closed, semi-closed wards compared to open wards), sex (commoner among female nurses), age and experience (older age and longer professional experience are associated with reduced frequency of experiencing aggressive acts). ${ }^{7}$ 
In Nigeria, the experience of nurses or other mental health professionals concerning in-patient aggression has not been explored. Several reports show that stigmatising and discriminatory dispositions held toward the mentally ill by lay persons are also held by health care professionals in the country. ${ }^{6,9-11}$ Health professionals view the mentally ill as a group easily prone to violence, unpredictability and often malevolence. ${ }^{11}$ Furthermore, the combination of high inpatient numbers and poor staffing in most psychiatric treatment units, may be associated with a high incidence of aggressive acts which often escalate when poorly handled. ${ }^{12}$ Against this backdrop, we conducted an exploratory study to determine mental health nurses' attitudes towards, and perception of, the prevalence of aggression within in-patient settings. This study was considered relevant in order to form a basis for the design and implementation of a locally relevant training program focused on the effective management of aggression in psychiatric units in particular and in other treatment settings in general.

\section{Method \\ Study setting}

The study was conducted at two centres: The Federal Psychiatric Hospital, Uselu (FPHU); and the University of Benin Teaching Hospital (UBTH), both in Benin City, Nigeria. The FPHU is a 220-bed stand-alone psychiatric referral facility. In-patient mental health services at the UBTH are provided from a 20-bed unit. Both facilities provide services to an estimated 8 million people from a catchment area covering 3 States of the country. At the FPHU, in-patient care is offered from 6 male wards, 4 female wards, a drug dependence ward and a child/adolescent ward, while at the UBTH a single ward is demarcated to serve male and female in-patients. All wards at both centres are run on a closed system. The drug dependence ward at the FPHU runs a semi-closed system.

\section{Respondents}

All mental health nursing staff at both study sites who had worked for at least a year at the time of the study were recruited. Pre-tested questionnaires were administered after the aim of the study had been explained and written informed consent obtained. Questionnaires were distributed and retrieved by one of the authors.

\section{Ethical clearance}

The study protocol was approved by the Ethical Clearance Committee of the Psychiatric Hospital, Uselu, Benin City, Edo State.

\section{Questionnaires}

The questionnaire comprised three sections:

Section A: A Socio-demographic Questionnaire: designed by the authors to obtain variables such as age, gender, religion, and duration of experience in mental health nursing

Section B: Attitudes toward aggression scale; ATAS ${ }^{13}$ : this is an 18-item self report scale designed for the assessment of staff attitudes toward in-patient aggression. The 18 statements on the ATAS comprise relevant themes on aggression with response options varying on a 5-point Likert scale from totally agree (5) to totally disagree (1). This scale comprises five sub-scales, viz: offensive attitude (seeing aggression as unpleasant, hurtful and an unacceptable behaviour -7 items); communicative attitude (aggression as a signal resulting from a patients powerlessness aimed at enhancing a therapeutic relationship - 3 items); destructive attitude (aggression as a threat or act of physical harm - 3 items); protective attitude (aggression as shielding or defending of physical and emotional space - 2 items) and intrusive attitude (viewing aggression as the expression to damage or injure others - 3 items). The ATAS has been shown to be a reliable and valid instrument providing stable measures across cultures. ${ }^{12-13}$ Higher scores on the 5 sub-scales indicate an increased likelihood of staff attitudes endorsing the particular attitude expressed by that subscale. In this study the ATAS, was found to be a fairly reliable questionnaire with a Cronbach's alpha of 0.76.

Section C: Prevalence of the perception of aggression scale; POPAS ${ }^{14}$ : The POPAS is a 16-item self report questionnaire that measures nurses' perceptions of the prevalence and types of aggression they have been confronted with or have witnessed during the course of the past year. For the purpose of our study, we made further modifications to a modified version 12-item version of the POPAS ${ }^{15}$ following pilot testing. Non-threatening and threatening verbal aggression were combined to constitute verbal aggression; provocative and passive aggressive behaviour were removed from the questionnaire because of their rarity and the difficulty experienced by nursing staff in the pilot group in discriminating it from threatening physical aggression. We produced a modified 9-item version of the POPAS for the purpose of this study: (1) Verbal aggression: shouting, cursing, yelling; (2) humiliating aggressive behaviour: expressing clear personal insults, abusive cursing, name calling, making discriminating remarks/gestures or spitting, all of which are perceived as making an impression and bringing down pride and self esteem; (3) aggressive splitting behaviour: behaviour that divides a group in two opposite poles; (4) threatening physical aggression: behaviour that is perceived as threatening; (5) destructive aggressive behaviour: behaviour that causes damage; (6) physical violence: behaviour that will harm or cause injury; (7) violence against self: mutilating behaviour against oneself, with minor or serious injury as a result; (8) suicide attempt; and (9) sexual intimidation/harassment: behaviour ranging from making obscene gestures to threatening with assault or rape. The response options ranged from (1) never to (5) frequently and the respondents were also asked to estimate the number of times they have had experiences with each type of aggression during the course of the past year at work. Number of days due to sick leave was also enquired, as well as number of these days due to aggression. The Cronbach's alpha for the modified POPAS used in this study was 0.70 , indicating good reliability. 


\section{Data analysis}

The data were analyzed using the Statistical Package for the Social Sciences (SPSS 17.0 for Windows). Descriptive analyses; percentages, means and standard deviations were used to characterize the socio-demographic characteristics of the sample population and determine the scores on the main variables including the sub-scales of the ATAS and the POPA.S. Bivariate analyses using independent sample t-tests and Mann-Whitney U test where appropriate were used to compare sex, age $(<38 / \geq 38$ years), duration of work experience $(<10 / \geq 10$ years) and subscales of the ATAS as well as types of aggression experienced on the POPAS. Statistical significance was set at $\mathrm{p}<0.05$.

\section{Results}

Of a total of 102 questionnaires sent out to mental health nurses at both study sites, 76 questionnaires were subsequently returned (75\% participation rate). Three questionnaires which were incompletely filled were discarded, leaving a total of 73 analysable questionnaires.

\section{Socio-demographic characteristics}

The respondents were predominantly female (71.2\%), Christian (95.9\%) and with a mean age ( \pm SD) of 38.78 ( \pm 7.55 years). Their ages ranged between 23 and 59 years, with the mean age of males significantly higher than that of females $(t=3.70, d f=71, p>0.001)$. The average duration of professional experience was $11.10( \pm 7.40)$ years. The duration of professional experience ranged from 1 to 32 years.

\section{Attitudes toward in-patient aggression}

The mean scores ( \pm SD) for the sample on each of the five subscales of the ATAS indicated that they considered inpatient aggression to be highly offensive; 26.16 ( \pm 4.58$)$, least communicative; 9.56 ( \pm 2.32$)$, destructive; 12.05 ( \pm 2.39$)$, not protective; 5.04 ( \pm 2.38 ), and intrusive; 9.86 $( \pm 2.56)$, as per the earlier description of the subscales (under Method-Questionnaires-Section B). A comparison of mean values of the component sub-scales of the ATAS with sex, showed that female nurses were more likely to view inpatient aggression as a means of communication compared to male nurses $(\mathrm{t}=-2.391, \mathrm{df}=71, \mathrm{p}<0.019)$.

\section{Prevalence and pattern of aggression experienced in the past year}

The perceived prevalence of various types of aggression by nurses in our sample is illustrated in Table I. Nurses were most likely to report experiencing in the past year verbal aggression, humiliating aggression, aggressive splitting behaviour, threatening physical aggression, destructive aggressive behaviour and physical violence occasionally or sometimes. Severe violence against self, suicide attempts and sexual intimidation were never or rarely experienced in the past year. Male nurses were significantly more likely to report experiencing episodes of physical violence compared to their female counterparts $(t=1.97, \mathrm{df}=71$, $p<0.019$ ). Nurses with less than 10 years of professional experience were more likely to report experiencing fewer episodes of humiliating aggression $(t=-2.08, \mathrm{df}=71$, $\mathrm{p}<0.038)$ and sexual intimidation $(\mathrm{t}=-2.40, \mathrm{df}=7 \mathrm{l}, \mathrm{p}<0.02)$.

\begin{tabular}{|c|c|c|c|}
\hline & Never & $\begin{array}{l}\text { Occasionally/ } \\
\text { sometimes }\end{array}$ & $\begin{array}{l}\text { Often/ } \\
\text { frequently }\end{array}$ \\
\hline 1. Verbal aggression & 0 & 67.2 & 32.8 \\
\hline 2. Humiliating aggression & 13.7 & 68.5 & 17.8 \\
\hline 3. Aggressive splitting behaviour & 34.2 & 61.6 & 4.1 \\
\hline 4. Threatening physical aggression & 12.3 & 72.6 & 15.1 \\
\hline 5. Destructive aggressive behaviour & 37.0 & 56.1 & 6.9 \\
\hline 6. Physical violence & 35.6 & 61.6 & 2.8 \\
\hline 7. Severe violence against self & 69.9 & 28.8 & 1.4 \\
\hline 8. Suicide attempts & 72.6 & 23.3 & 4.1 \\
\hline 9. Sexual intimidation/harassment & 67.1 & 31.5 & 1.4 \\
\hline
\end{tabular}

\begin{tabular}{|c|c|c|}
\hline & Range & Mean $( \pm S D)$ \\
\hline 1. Verbal aggression & $0-1,000$ & $48.23(35.86)$ \\
\hline 2. Humiliating aggression & $0-360$ & $23.93(59.38)$ \\
\hline 3. Aggressive splitting behaviour & $0-26$ & $3.12(4.90)$ \\
\hline 4. Threatening physical aggression & $0-1,000$ & $26.73(118.78)$ \\
\hline 5. Destructive aggressive behaviour & $0-100$ & $9.64(25.01)$ \\
\hline 6. Physical violence & $0-24$ & $2.96(4.69)$ \\
\hline 7. Severe violence against self & $0-10$ & $0.97(2.25)$ \\
\hline 8. Suicide attempts & $0-10$ & $0.96(1.94)$ \\
\hline 9. Sexual intimidation/harassment & $0-10$ & $0.96(1.97)$ \\
\hline
\end{tabular}

There was wide variability in the number of episodes of various types of aggression recorded by the nurses (Table II). As a result, in terms of actual estimated numbers of types of aggression seen in the past year, nurses with over 10 years of professional experience reported significantly higher numbers of episodes of physical aggression (Mann Whitney $\mathrm{U}=468.000, \mathrm{p}<0.05$, 2-tailed) and humiliating aggression (Mann Whitney U=422.00, p<0.014, 2-tailed). Male nurses reported experiencing significantly higher number of episodes of aggressive spitting behaviour (Mann Whitney U=341.50, p<0.01 1, 2-tailed) as well as physical violence (Mann Whitney U=340.50, p<0.010, 2-tailed).

\section{Sick leave}

A minority 24 (32.9\%) of the sample had required sick leave in the year prior to the commencement of the study. The number of days spent on sick leave, ranged between 1-5 days, with an average duration of $0.68( \pm 1.77)$ days. Furthermore, only 5 (9.9\%) had required sick leave due to acts of aggression exhibited by patients. Sick leave days due to aggression varied from 1-3 days.

\section{Discussion}

To the knowledge of the authors, this is the first study to examine the attitudes of nurses towards aggression by psychiatric in-patients in Nigeria. This study found that nurses in Nigeria were more inclined to perceiving aggression as offensive, destructive and intrusive. They were less inclined to view it as communicative or as an 
action with protective goals. The attitudes of nurses in this study mirror that in the study conducted by Jonker and his colleagues ${ }^{7}$ in the Netherlands and contrasts with findings from an earlier study by Jansen et al. ${ }^{13}$ in the same country where aggression was viewed as largely communicative and protective. As we had theorized, the large proportion of patients admitted into psychiatric units involuntarily, as well as the practice of operating a closed-ward system at the study sites, are possible factors accounting for a high degree of intolerance by Nigerian nurses for psychiatric inpatient aggression. The tendency for nurses in this country to view aggression as largely negative has several implications for patient care. Firstly, patients who exhibit acts of aggression are more likely to receive poorer care. At the study sites, physical restraints and sedation using psychopharmacological agents are the commonest methods of managing aggression. As a result of understaffing, less coercive means of controlling aggression are rarely employed until acts of aggression have escalated resulting in unrestrained use of chemical sedation and/or physical restraints. Secondly, a negative attitude is likely to predispose staff to exhibit actions that may precipitate severe acts of aggression from patients. These actions which might include coercing patients to take routine medications, restricting in-patient ward activity or patient interaction might act as unintended triggers of aggressive acts. Female nurses in this study were more likely to view aggression as having a communicative role. It remains to be seen if the tilt towards a higher representation of the female gender in our sample would account for lower numbers of types of aggression reported.

The pattern of aggressive acts reported in this study varied from the very common (verbal aggression) to the least occurring (suicide attempts and sexual intimidation). Similar findings were observed in studies conducted in the Netherland.s. ${ }^{7,14}$ Longer professional experience was significantly associated with higher frequency of physical violence as well as episodes of aggressive splitting behaviour. Furthermore male nurses reported experiencing higher episodes of physical aggression and aggressive splitting behaviour compared to their female counterparts. A possible explanation for these findings would include the possibility that more experienced staff who are intolerant of aggression would more likely engage patients who exhibit mild acts of aggression and, with a negative predisposition to these acts, encounters could escalate. Male nurses are more likely to be involved or called upon by their female counterparts to mediate in calming aggressive patients with the result that they are more exposed to violent acts. Though aggressive acts are likely to occur more frequently in closed wards, where a majority of patients are admitted involuntarily, the frequency of different types of aggression reported in this study was lower when compared to similar studies. ${ }^{7,14-15}$ Perhaps as posited in the paper by Jonker et al. ${ }^{7}$, aggressive acts now occur commonly such that nursing staff have become insensitive to the frequency of their occurrence and now see them as routine. A reflection of this view may be inferred from the small proportion of the sample that required sick leave due to acts of aggression. This number was also much lower than that reported by Nijman and colleagues. ${ }^{15}$
The current study is not without its limitations. Though the modified form of the POPAS and the ATAS showed fairly good reliability, testing the validity of the instruments in this cultural environment was not undertaken. We relied on face validity alone. Secondly, our sample size is moderate and from only two psychiatric facilities in the country. This might limit the ability to generalise of the results.

\section{Conclusion}

This study found that aggression is negatively viewed by Nigerian psychiatric nurses. These attitudes are reflective of the opinions of lay persons in our society. There is a need for training programs to reorient the opinions of nurses in relation to inpatient aggression. These programs should contribute towards improved patient care and reduction in the frequency of aggressive acts within in-patient units.

\section{References}

1. Bouras N, Trauter T, Watson JP. Ward environment and disturbing behaviour. Psychological Medicine 1982; 12:309-319.

2. Irwin $A$. The nurse's role in the management of aggression. Journal of Psychiatric and Mental Health Nursing 2006; 13:309-318.

3. Jansen GJ, Dassen TW, Groot Jebbink G. Staff attitudes towards aggression in health care: a review of the literature. Journal of Psychiatric and Mental Health Nursing 2005; 12:3-13.

4. Jansen GJ, Middel LJ, Dassen TWN. An international comparative study on the reliability and validity of the Attitudes towards Aggression Scale (ATAS). International Journal of Nursing Studies 2005; 42:467-477.

5. Zimbardo P, Ebbesen EB. Influencing attitudes and changing behaviour. Reading, Mass: Addison-Wesley 1969;567-606.

6. Ewhrudjakpor C. Knowledge, beliefs and attitudes of health care providers towards the mentally ill in Delta State, Nigeria. Studies on Ethno-Medicine 2009; 3:19-25

7. Jonker EJ, Goossens PJJ, Steenhuis IHM, Oud NE. Patient aggression in clinical psychiatry: perceptions of mental health nurses. Journal of Psychiatric and Mental Health Nursing 2008; 15:492-499.

8. Finnema EJ, Dassen T, Halfens R. Aggression in psychiatry: a qualitative study focusing on the characterization and perception of patient aggression by nurses working on psychiatric wards. Journal of Advanced Nursing 1994; 19:1088-1095.

9. Aghukwa NC. Attitude of health workers to the care of psychiatric patients. Annals of General Psychiatry 2009; 8: 19.

10. Mohammed Z, Isa SA, Muktar HA. Perception and beliefs about mental illness among Karfi village, Northern Nigeria. Journal of International Health and Human Rights 2004; 4:1.

11. Gureje O, Lasebikan V, Oluwanuga OE, Olley BO, Kola L. Community study of knowledge and attitude to mental illness in Nigeria. British Journal of Psychiatry 2005; 186:436-441.

12. Gureje O. Psychiatry in Nigeria. International Psychiatry 2003; 2:1011.

13. Jansen GJ, Dassen TW, Burgehof J. Psychiatric nurses' attitudes toward inpatient aggression: preliminary report of the development of the attitude towards aggression scale (ATAS). Aggressive Behaviour 2006; 32:44-53.

14. Oud N. Internal report. POPAS Ervaringen van psychiatrische hulpverleners met agressief gedrag 2001; 1-15.

15. Nijman H, Bowers L, Oud N, Jansen G. Psychiatric nurses' experiences with inpatient aggression. Aggressive Behaviour 2005; 31:217-227. 